\title{
Bulk diffusivity of lattice gases close to criticality
}

\section{Citation}

Spohn, Herbert, and Horng-Tzer Yau. 1995. "Bulk Diffusivity of Lattice Gases Close to Criticality." Journal of Statistical Physics 79 (1-2) (April): 231-241. doi:10.1007/bf02179388.

\section{Published Version}

doi:10.1007/BF02179388

\section{Permanent link}

http://nrs.harvard.edu/urn-3:HUL.InstRepos:32706721

\section{Terms of Use}

This article was downloaded from Harvard University's DASH repository, and is made available under the terms and conditions applicable to Other Posted Material, as set forth at http:// nrs.harvard.edu/urn-3:HUL.InstRepos:dash.current.terms-of-use\#LAA

\section{Share Your Story}

The Harvard community has made this article openly available.

Please share how this access benefits you. Submit a story.

\section{Accessibility}




\title{
Bulk Diffusivity of Lattice Gases Close to Criticality
}

\author{
Herbert Spohn*, Horng-Tzer Yau ${ }^{\dagger}$ \\ Courant Institute, New York University \\ New York, NY 10012 \\ e-mail: yau@math.nyu.edu
}

\begin{abstract}
We consider lattice gases where particles jump at random times constrained by hard core exclusion (simple exclusion process with speed change). The conventional theory of critical slowing down predicts that close to a critical point the bulk diffusivity vanishes as the inverse compressibility. We confirm this claim by proving a strictly positive lower bound for the conductivity.
\end{abstract}

\section{Introduction}

Close to a critical point dynamical processes become sluggish. Such a critical slowing down can be infered already from the most primitive approximation. E.g. if the order parameter $m$ is supposed to satisfy $m=-V^{\prime}(m)$ with $V(m)=a m^{2}+b m^{4}, b>0$, then as $a \rightarrow 0_{+}$ the relaxation to the equilibrium point $m=0$ becomes slow. A more demanding problem is to extract such a slow behavior out of a microscopic model with many degrees of freedom. Now, the conventional theory [1,2] asserts that dynamical processes slow down because close to criticality certain thermodynamic susceptibilities diverge. No extra complications are supposed to arise from the dynamics itself. In fact, the conventional theory turns out to

*permanent address: Theoretische Physik, Theresienstr. 37, 80333 München, Germany

${ }^{\dagger}$ Research partially supported by U.S. National Science Foundation grant 9101196, Sloan Foundation Fellowship and David and Lucile Packard Foundation Fellowship. 
be wrong for an Ising model with spin flip dynamics, at least below the upper critical dimension. There is then an independent dynamical scaling exponent governing the slow decay at $T_{c}[3]$. On the other hand for a conserved field a renormalization group calculation supports the conventional theory $[3,4]$. There has been considerable numerical effort to verify these predictions, cf. [5], e.g.. Since for lattice gases close to criticality there is slowing down on top of a conservation law, Monte Carlo simulations are plagued by substantial numerical uncertainties. To our knowledge, the most extensive Monte Carlo computation [6] obtains results at least consistent with the conventional theory.

The aim of our paper is to prove bounds on the bulk diffusivity, $D(\rho)$, as

$$
d_{-} \rho(1-\rho) \chi(\rho)^{-1} \leq D(\rho) \leq d_{+} \rho(1-\rho) \chi(\rho)^{-1} .
$$

Here $\rho$ is the density of the lattice gas, $0 \leq \rho \leq 1, \chi(\rho)$ is its compressibility, and $0<d_{-} \leq$ $d_{+}<\infty$ are suitable constants independent of the density. Thus, if $\chi(\rho)=\left|\rho-\rho_{c}\right|^{-(\delta-1)}, \delta>$

1 , close to the critical density $\rho_{c}$, then $D(\rho)$ vanishes as $\left|\rho-\rho_{c}\right|^{(\delta-1)}$ for $\rho \rightarrow \rho_{c}$. This is precisely one of the predictions of the conventional theory.

Our paper is organized as follows: In Section 2 we briefly recall the definition of lattice gases, explain in more detail the claims of the conventional theory, and define the bulk diffusivity. In Section 3 we establish a lower bound on the bulk diffusivity, the upper bound in (1.1) being trivial.

\section{Conventional Theory and Bulk Diffusivity}

We consider a lattice gas on a simple hypercubic lattice $Z^{d}$. Although the bound to be established holds in fair generality (see remarks below), for the sake of concreteness and notational simplicity we restrict ourselves to the nearest neighbor case. As standard, the occupation variables are denoted by $\eta(x), \eta(x)=0,1$ with $x \in \mathbb{X}^{d}, \Omega$ and a whole particle configuration is denoted by $\eta: X^{d} \rightarrow\{0,1\}$. The dynamics is governed by the exchange rates $c(x, y, \eta)$. We require $c(x, y, \eta)=0$ unless $|x-y|=1$ and to be nondegenerate in the sense

$$
0<c_{-} \leq c(x, y, \eta) \leq c_{+}<\infty
$$


for $|x-y|=1$. The rates $c(x, y)$ are assumed to depend on $\eta$ only through $\{\eta(z):|x-z|<$ $\left.R_{0},|y-z|<R_{0}\right\}, 1 \leq R_{0}<\infty$, to be translation invariant, namely $c(x, y, \eta)=c(x+a, y+$ $\left.a, \tau_{a} \eta\right)$ with $\tau_{a}$ the shift by $a$, and to be invariant under lattice rotations. The generator for the dynamics is then given by

$$
\mathcal{L} f(\eta)=\sum_{<x, y>} c(x, y, \eta)\left[f\left(\eta^{x y}\right)-f(\eta)\right]
$$

acting on local functions $f$. Here $\langle x, y\rangle$ denotes a pair of nearest neighbors and

$$
\eta^{x y}(z)= \begin{cases}\eta(y), & \text { if } z=x, \\ \eta(x), & \text { if } z=y, \\ \eta(z), & \text { if } z \neq x, y .\end{cases}
$$

$\mathcal{L}$ generates the Markov semigroup $\exp [\mathcal{L} t]$ on $C\left(\{0,1\}^{X^{d}}\right)$ and there exists a corresponding stochastic jump process $\eta_{t}$, cf. [7] for details.

We require our dynamics to be reversible. For this purpose we define the energy

$$
H(\eta)=-\beta \sum_{<x, y>} \eta(x) \eta(y)
$$

$\beta>0$ is an attractive and $\beta<0$ a repulsive lattice gas. For a bond $(x, y)$ let $\Delta_{x y} H(\eta)=$ $H\left(\eta^{x y}\right)-H(\eta)$ be the energy difference. We impose then the condition

$$
c(x, y, \eta)=c\left(x, y, \eta^{x y}\right) e^{-\Delta_{x y} H(\eta)} .
$$

In particular, Eq. (2.4) implies that the set of canonical Gibbs measures with the nearest neighbor potential $J_{\{x, y\}}=-\beta \eta(x) \eta(y)$ is invariant and reversible under $\mathcal{L}$ [8]. We denote a translation invariant (not necessarily extreme) canonical Gibbs measure by $\mu_{\rho}$ and its expectations by $\langle\cdot\rangle_{\rho}$, where the subscript $\rho$ labels the average density, $\langle\eta(x)\rangle_{\rho}=\rho$. The stochastic process $\eta_{t}, t \in \boldsymbol{R}$, with initial measure $\mu_{\rho}$ is space-time stationary. Expectations with respect to this process are denoted by $E^{\rho}$.

The Gibbs measures will play a prominent role and it might be useful to first describe their phase diagram (for $d \geq 2$ ) as presented in Figure 1 . 
We plot the average density against $\beta$ (not to scale). In region I there is a unique Gibbs measure. In region II, we have two extremal Gibbs measures which transform into each other by a unit shift. Their typical configurations have a checkerboard pattern. The shaded regions III and IV correspond to mixtures of the Gibbs measures living at the boundary points of the density interval with $\beta$ fixed. $\left(\beta_{c}, \frac{1}{2}\right)$ is the critical point in the attractive case. For $-\beta_{t}<\beta \leq-\beta_{c}$, there is a line of critical points which terminates in two critical endpoints $[9,10]$. Only some parts of the phase diagram have been established mathematically $[11,12,13]$.

We define the static compressibility by

$$
\chi(\rho)=\sum_{x \in \mathbb{Z}^{d}}\left(<\eta(x) \eta(0)>_{\rho}-\rho^{2}\right)
$$

for $\rho$ in region I and II. For $\beta=\beta_{c}, \chi(\rho)$ diverges as $\rho \rightarrow \frac{1}{2}$. Similarly, for $-\beta_{t}<\beta<-\beta_{c}$ and $d=2,3, \chi(\rho)$ diverges as $\rho$ approaches the line of critical points.

Our real interest is the dynamics. The most basic property is the spreading of a density disturbance in equilibrium. As a quantitative measure one adopts the bulk diffusivity, $D$. Since our lattice gas is isotropic by assumption, $D$ is a scalar and can be defined through the normalized second moment as

$$
D=\lim _{t \rightarrow \infty} \frac{1}{2 d t} \chi^{-1} \sum_{x \in \mathbb{Z}^{d}} x^{2}\left[E^{\rho}\left(\eta_{t}(x) \eta_{0}(0)\right)-\rho^{2}\right] \equiv \chi^{-1} \sigma
$$

If $\mu_{\rho}$ satisfies an exponential mixing condition, then the limit (2.6) exists and $\sigma$ is given through the variational formula [14]

$$
\sigma=\inf _{G} \frac{1}{2} \sum_{j=1}^{d}<c\left(0, e_{j}\right)\left[\left(e_{1} \cdot e_{j}\right)\left(\eta(0)-\eta\left(e_{j}\right)\right)+D_{0 e_{j}} \sum_{x \in \mathcal{Z}^{d}} \tau_{x} G\right]^{2}>_{\rho} .
$$

The infimum is over all local functions $G$. Here $e_{j}$ is the unit vector along the positive $j$ axis and the exchange operator is defined by

$$
D_{x y} f(\eta)=f\left(\eta^{x y}\right)-f(\eta)
$$

Note that the sum over $\mathbb{Z}^{d}$ contains only a finite number of non-zero terms. Physically $\sigma$ has the meaning of a conductivity, i.e., if the exchange rates are slightly biased along the 1-axis, 
then a steady state current is induced which turns out to be proportional to $\sigma$ [14]. From now on, we regard Eq. (2.7) as the definition of $\sigma$.

Taking $G=0$ in (2.7) yields the upper bound

$$
\sigma \leq c(\rho) d_{+}
$$

with $d_{+}=c_{+} / 2$ and $c(\rho)=<\left(\eta(0)-\eta\left(e_{1}\right)\right)^{2}>_{\rho}$. The conventional theory postulates that $\sigma$ remains strictly positive close to a critical point. By $(2.6)$ this would imply that $D(\rho)$ must vanish as $\chi(\rho)^{-1}$ for $\rho \rightarrow \rho_{c}$. At first sight this claim looks rather innocent. However along with it goes a prediction on the scaling close to $\rho_{c}$ as we explain now. We define the structure function

$$
\widehat{S}(k, t)=\sum_{x \in \mathcal{Z}^{d}} e^{i k \cdot x}\left(E^{\rho}\left(\eta_{t}(x) \eta_{0}(0)\right)-\rho^{2}\right)
$$

with $\rho$ in region I and II. The conventional theory argues that for small $k$ and large $t$

$$
\widehat{S}(k, t) \cong \widehat{S}(k, 0) \exp \left[-\sigma k^{2}|t| / \widehat{S}(k, 0)\right]
$$

Away from criticality $\chi=\lim _{k \rightarrow 0} \widehat{S}(k, 0)$ and we recover the standard diffusive spreading of a density disturbance with diffusion coefficient $D=\sigma / \chi$. However, at criticality $\widehat{S}(k, 0) \cong$ $c_{0}|k|^{-2+\eta}$ for $k \rightarrow 0$. If $\sigma$ does not vanish, then at the critical point

$$
\widehat{S}(k, t) \cong c_{0}|k|^{-2+\eta} \exp \left[-\left(\sigma / c_{0}\right)|k|^{4-\eta}|t|\right]
$$

The scaling form (2.11) is somewhat remote from rigorous analysis. Our goal here is more modest.

Theorem 1. Let $\Lambda$ be a box with periodic boundary conditions. Let $\mu_{\rho, \Lambda}$ be the Gibbs measure $Z^{-1} \exp \left[\beta \sum_{<x, y>, x, y \in \Lambda} \eta(x) \eta(y)\right]$, constrained to the set $\left\{\eta \in\{0,1\}^{\Lambda}:[\rho|\Lambda|]=\right.$ $\left.\sum_{x \in \Lambda} \eta(x)\right\}, 0<\rho<1$, where [.] denotes the integer part and $|\Lambda|$ the number of sites in $\Lambda$. Let $\sigma$ be defined by the variational form (2.7) with $\mu_{\rho}$ any weak limit point of the sequence $\mu_{\rho, \Lambda},|\Lambda| \rightarrow \infty$. There exists then a constant $d_{-}>0$ such that

$$
c(\rho) d_{-} \leq \sigma
$$

with $c(\rho)=<\left(\eta(0)-\eta\left(e_{1}\right)\right)^{2}>_{\rho}$. 
Remarks (1) In the context of Ginzburg-Landau models Theorem 1 can easily be proved using the Schwarz inequality [16].

(2) The bound $d_{-}$is fairly explicit, cf. Eqs. (3.1), (3.10), but not too precise. It basically reflects that at low temperature the time scale for diffusion increases as $\exp [2 d|\beta|]$.

(3) $\chi(\rho)=\rho$ as $\rho \rightarrow 0$ and $\chi(\rho)=1-\rho$ for $\rho \rightarrow 1$. $c(\rho)$ has the same limit behavior. Therefore $D(\rho)$ tends to a non-zero value as $\rho \rightarrow 0, \rho \rightarrow 1$.

(4) The proof of Theorem 1 goes through whenever the potential for the Gibbs measure and the exchange rates are of finite range.

(5) Let $\beta>\beta_{c}$. Then in region III we have $\rho=\lambda \rho_{-}+(1-\lambda) \rho_{+}, 0<\lambda<1$. Correspondingly $\mu_{\rho}=\lambda \mu_{\rho_{-}}+(1-\lambda) \mu_{\rho_{+}}, \sigma(\rho)=\lambda \sigma\left(\rho_{-}\right)+(1-\lambda) \sigma\left(\rho_{+}\right)$. On the other hand density fluctuations are macroscopic (proportional to $|\Lambda|$ ) and therefore $\chi(\rho)=\infty$ for $\rho_{-}<\rho<\rho_{+}$. Since $\chi(\rho)^{-1}$ jumps from a non-zero value to zero at $\rho_{+}$and $\rho_{-}, D(\rho)$ is bounded away from zero for $0 \leq \rho \leq \rho_{-}$or $\rho_{+} \leq \rho \leq 1$ and vanishes for $\rho_{-}<\rho<\rho_{+}$. The same argument applies to region IV. Thus in the shaded regions we have $D(\rho)=0$. [In the context of Ginzbug-Landau models Rezakhanlou [15] proves that this extension into the two phase region is indeed the correct one.]

(6) By the spectral representation and Jensen inequality one has the lower bound $\widehat{S}(k, t) \geq$ $\widehat{S}(k, 0) \exp \left[-\sigma_{0} k^{2}|t| / \widehat{S}(k, 0)\right]$, compare with Eq. (2.10). Here $\sigma_{0} \geq \sigma$ and $\sigma_{0}$ is given by taking $G=0$ in $(2.7)$.

Addendum Although somewhat off the main track, the reader might be curious to know how $D(\rho)$ vanishes as $\rho \rightarrow \rho_{c}$ according to current knowledge. To facilitate comparison with the literature we use standard symbols for the critical exponents. In $d=2$ most of them come from the Onsager solution. Above the upper critical dimension one has mean field values. In between one has a variety of approximate methods. Three cases have to be distinguished. (1) $\beta=\beta_{c}, \rho_{c}=\frac{1}{2}$. The order parameter field and the conserved field agree. This is known as Model B in critical dynamics. The scaling form for the structure function is written as $|k|^{-2+\eta} \exp \left[-|k|^{z}|t|\right]$ with $z=4-\eta=2+(2-\eta)$. (ii) $-\beta_{t}<\beta<-\beta_{c}, \rho_{c}=\rho_{c}(\beta)$. The order parameter field is the staggered density which differs from the conserved field. This is known as Model $\mathrm{C}$ in critical dynamics. The scaling form for the structure function 
is written as $|k|^{-\alpha / \nu} \exp \left[-|k|^{z}|t|\right]$ with $z=2+\alpha / \nu$. (iii) $\beta=-\beta_{t}, \rho=\rho_{t}$. This is a tricritical point. The notation is as in (ii) with index $t$.

ad (i): One has $D(\rho) \cong\left|\rho-\frac{1}{2}\right|^{\delta-1}$ with $\delta-1=14$ for $d=2, \delta-1 \cong 3.8$ for $d=3$, and $\delta-1=2$ for $d \geq 4$. Also $\eta=\frac{1}{4}$ for $d=2, \eta \cong 0.03$ for $d=3$, and $\eta=0$ for $d \geq 4$.

ad (ii): $\beta_{c}(\rho)$ is parabolic close to $\rho=\frac{1}{2}$. One has $D(\rho) \cong\left|\rho-\rho_{c}\right|^{\alpha /(1-\alpha)}$ with $D$ vanishing as an inverse logarithm in $d=2, \alpha /(1-\alpha) \cong 0.12$ for $d=3$, and $D$ bounded for $d \geq 4$. At $\beta=-\beta_{c}, D$ remains bounded. The structure function diverges logarithmically, for $d=2$ diverges as $|k|^{-0.17}$ for $d=3$, and remains bounded for $d \geq 4$, cf. [9].

ad (iii): One has $D(\rho) \cong\left|\rho-\rho_{t}\right|^{\alpha_{t} /\left(1-\alpha_{t}\right)}$ with $\alpha_{t} /\left(1-\alpha_{t}\right)=8$ for $d=2$ and $\alpha_{t} /\left(1-\alpha_{t}\right)=1$ for $d \geq 3$, cf. [9].

\section{A Lower Bound}

We prove Theorem 1. Let $\Lambda=[0,2 \ell]^{d} \subset Z^{d}$ with $\ell>0$ and integer. We make out of $\Lambda$ a torus by considering $(\ldots, 2 \ell, \ldots)$ and $(\ldots, 0, \ldots)$ as nearest neighbors (= periodic boundary conditions). Let $\ell>R_{0}$. Then $c(x, y)$ is defined for every bond on the torus $\Lambda$. Let $<\cdot>_{\Lambda}$ denote expectation with respect to the Gibbs measure $Z^{-1} e^{-H}$ on the torus $\Lambda$ constrained to the set $\left\{\eta \in\{0,1\}^{\Lambda}: \sum_{x \in \Lambda} \eta(x)=[\rho|\Lambda|]\right\}$. We write $x=\left(x_{1}, x_{-}\right)$where $x_{1} \in \mathbb{Z}$ is along the 1 -axis and $x_{-} \in X^{d-1}$ is orthogonal to the 1 -axis.

We first prove a lemma which states that long range jumps can be replaced by short range jumps. This lemma is well known and can be proved with standard method if the inverse temperature $\beta=0$, namely for the identical independent random variables. In [16], this lemma was extended to the independent but not necessary identical case. The method we employ here uses idea similar to [16].

Lemma 2. For every function $u$ on $\{0,1\}^{|\Lambda|}$ we have

$$
\frac{1}{2 \ell}<\left[D_{(0,0)(2 \ell, 0)} u\right]^{2}>_{\Lambda} \leq \quad c_{0} \sum_{y=0}^{2 \ell-1}<\left[D_{(y, 0)(y+1,0)} u\right]^{2}>_{\Lambda}
$$

with $c_{0}=\left(2 e^{4 d|\beta|}\right)^{2 d+2}$. 
Proof: Let $A=\{(0,0),(2,0), \ldots,(2 \ell, 0)\} \subset \Lambda$. For notational simplicity we label these lattice sites by $j=0, \ldots, \ell$. We condition on $\eta^{c}=\{\eta(x) \mid x \in \Lambda \backslash A\}$. Because $H$ is nearest neighbor, the conditional measure is of the form

$$
\left(\prod_{j=0}^{\ell} p_{j}\left(\eta(j) \mid \eta^{c}\right)\right) h\left(\sum_{j=0}^{\ell} \eta(j), \eta^{c}\right)
$$

Expectations with respect to this measure are denoted by $<\cdot>_{\eta^{c}}$. The function $h$ ensures the global constraint on the density and the Gibbs factor read

$$
p_{j}\left(\eta(j) \mid \eta^{c}\right)=\left(\exp \left[\eta(j) E_{j}\left(\eta^{c}\right)\right]+\exp \left[(\eta(j)-1) E_{j}\left(\eta^{c}\right)\right]\right)^{-1}
$$

where $E_{j}$ takes only values in $\{0, \beta, \ldots, \beta 2 d\}$. Accordingly we partition $A$ into the $2 d+1$ disjoint sets $A_{r}=\left\{j \in A: E_{j}=\beta r\right\}, r=0,1, \ldots, 2 d$. The basic idea is to perform exchanges first only within $A_{0}$, then within $A_{1}$, etc..

We start with $A_{0}$ and label $A_{0}=\left\{y_{j}: j=1, \ldots, n\right\}, y_{j}<y_{j+1},\left|A_{0}\right|=n$. We also set $y_{0}=0$ and $y_{n+1}=\ell$, provided $y_{n}<\ell$. Let $T_{x y} u(\eta)=u\left(\eta^{x y}\right)$. Then

$$
T_{0 \ell}=T_{y_{0} y_{1}} \ldots T_{y_{n} y_{n+1}} T_{y_{n-1} y_{n}} \ldots T_{y_{0} y_{1}} .
$$

If either $y_{1}=0$ or $y_{n}=\ell$, then the corresponding factors are omitted. We write the telescoping sum

$$
D_{0 \ell} u=T_{y_{0} y_{1}} \ldots\left(T_{y_{0} y_{1}} u-u\right)+T_{y_{0} y_{1}} \ldots\left(T_{y_{2} y_{1}} u-u\right)+\ldots+\left(T_{y_{0} y_{1}} u-u\right)
$$

Now $\left(T_{y_{j} y_{j+1}} f\right)^{2}=T_{y_{j} y_{j+1}} f^{2}$ and $<T_{y_{j} y_{j+1}} f>_{\eta^{c}}=<f>_{\eta^{c}}$ provided $1 \leq j \leq n-1$. For the endpoints we use

$$
<T_{y_{0} y_{1}}|f|>_{\eta c} \leq e^{2 d|\beta|}<|f|>_{\eta^{c}},<T_{y_{n} y_{n+1}}|f|>_{\eta^{c}} \leq e^{2 d|\beta|}<|f|>_{\eta^{c}}
$$

Then, using (3.2) and Schwarz inequality, we arrive at

$$
\ell^{-1}<\left(D_{0 \ell} u\right)^{2}>_{\eta^{c}} \leq 2 e^{4 d|\beta|} \sum_{j=0}^{n}\left(y_{j+1}-y_{j}\right)^{-1}<\left(D_{y_{j} y_{j+1}} u\right)^{2}>_{\eta^{c}}
$$

If either $y_{1}=0$ or $y_{n}=\ell$, then the corresponding summands in (3.3) have to be ommitted. Note that terms on the right hand side of (3.3) are normalized by the jump length just as 
on the right. Thus whenever $y_{j+1}-y_{j}>1$ we may iterate our procedure for each isolated interval separately, now employing the subset $A_{1}$ instead of $A_{0}$ etc.. Then

$$
\ell^{-1}<\left(D_{0 \ell} u\right)^{2}>_{\eta^{c}} \leq\left(2 e^{4 d|\beta|}\right)^{2 d+1} \sum_{j=0}^{\ell-1}<\left(D_{j j+1} u\right)^{2}>_{\eta^{c}} .
$$

We average over $\eta^{c}$ and use that

$$
<\left(D_{x, x+2 e_{1}} u\right)^{2}>_{\Lambda} \leq 4 e^{4 d|\beta|}\left[<\left(D_{x, x+e_{1}} u\right)^{2}>_{\Lambda}+<\left(D_{x+e_{1}, x+2 e_{1}} u\right)^{2}>_{\Lambda}\right]
$$

Inserting this bound in Eq. (3.4) yields (3.1).

Proof of Theorem 1: We define

$$
W=\sum_{x_{-} \in[0,2 \ell]^{d-1}}\left(1+\exp \left[-\Delta_{\left(0, x_{-}\right)\left(2 \ell, x_{-}\right)} H\right]\right)\left(\eta\left(0, x_{-}\right)-\eta\left(2 \ell, x_{-}\right)\right)
$$

and

$$
\mathcal{L}=\sum_{\substack{<x, y>\\ x, y \in \Lambda}}{ }^{*} c(x, y) D_{x, y} .
$$

$\sum^{*}$ means that we omit all bonds in $\Lambda$ with end points $\left(2 \ell, x_{-}\right),\left(0, x_{-}\right)$and correspondingly for the other coordinate axes. Clearly, for any $h$ we have $<W h\left(\sum_{x \in \Lambda} \eta(x)\right)>_{\Lambda}=0$. Thus $W$ is orthogonal to the zero subspace of $\mathcal{L}$ and

$$
<W(-\mathcal{L})^{-1} W>_{\Lambda}=\sup _{u}\left\{2<W u>_{\Lambda}-\mathcal{D}(u)\right\}
$$

where $\mathcal{D}$ is the Dirichlet form for $\mathcal{L}$. We set $u=\alpha u^{*}$ with $\alpha \in \boldsymbol{R}$ and

$$
u^{*}=\sum_{x \in \Lambda}\left(e_{1} \cdot x\right) \eta(x)+\sum_{x \in \Lambda} \tau_{x} G
$$

Here $G$ is a function of finite range $R, \ell>R$, and $\tau_{x}$ is considered as a shift on the torus $\Lambda$. To compute the two terms in Eq. (3.7) we use the translation invariance $(\bmod \Lambda)$ of $<\cdot>_{\Lambda}$. We then obtain

$$
<W(-\mathcal{L})^{-1} W>_{\Lambda} \geq \sup _{\alpha}\left\{(2 \ell+1)^{d-1} 2 \ell\left[-2 \alpha \tau-2 \alpha^{2} \sigma_{\Lambda}(G)\right]+2 \alpha \gamma(G)\right\}
$$

with

$$
\tau=<(\eta(0,0)-\eta(2 \ell, 0))^{2}>_{\Lambda},
$$




$$
\begin{gathered}
\gamma(G)=\sum_{x \in \Lambda}<W \tau_{x} G>_{\Lambda}, \\
\sigma_{\Lambda}(G)=\frac{1}{2} \sum_{j=1}^{d}<c\left(0, e_{j}\right)\left[\left(e_{1} \cdot e_{j}\right)\left(\eta(0)-\eta\left(e_{j}\right)\right)+D_{0 e_{j}} \sum_{x} \tau_{x} G\right]^{2}>_{\Lambda} .
\end{gathered}
$$

Taking the supremum over $\alpha$ yields

$$
\sigma_{\Lambda}(G) \geq \frac{1}{2}(2 \ell+1)^{d-1} 2 \ell\left[\tau-(2 \ell+1)^{-d+1}(2 \ell)^{-1} \gamma(G)\right]^{2}<W(-\mathcal{L})^{-1} W>_{\Lambda}^{-1}
$$

We return to Eq. (3.7). By Schwarz inequality and Lemma 2

$$
\begin{gathered}
2\left|<W u>_{\Lambda}\right|=2\left|\sum_{x_{-} \in[0,2 \ell]^{d-1}}<\left[u\left(\eta^{\left(0, x_{-}\right)\left(2 \ell, x_{-}\right)}\right)-u(\eta)\right]\left[\eta\left(0, x_{-}\right)-\eta\left(2 \ell, x_{-}\right)\right]>_{\Lambda}\right| \\
\leq \sum_{x_{-} \in[0,2 \ell]^{d-1}}\left(c_{0} 2 \ell\right)^{-1}<\left[u\left(\eta^{\left(0, x_{-}\right)\left(2 \ell, x_{-}\right)}\right)-u(\eta)\right]^{2}>_{\Lambda} \\
+2 c_{0} \ell<\left[\eta\left(0, x_{-}\right)-\eta\left(2 \ell, x_{-}\right)\right]^{2}>_{\Lambda} \\
\leq \mathcal{D}(u)+c_{0}(2 \ell+1)^{d-1} 2 \ell \tau .
\end{gathered}
$$

Thus

$$
<W(-\mathcal{L})^{-1} W>_{\Lambda} \leq c_{0}(2 \ell+1)^{d-1} 2 \ell \tau
$$

and

$$
\sigma_{\Lambda}(G) \geq \frac{1}{2 c_{0}} \frac{1}{\tau}\left[\tau-(2 \ell+1)^{-d+1} 2 \ell^{-1} \gamma(G)\right]^{2}
$$

We now take a sequence of $\ell \rightarrow \infty$ such that $\mu_{\Lambda} \rightarrow \mu_{\rho}$ weakly. It follows that $|\gamma(G)| \leq$ const. $\ell^{d-1}$, since only those terms contribute to the sum where the support of $\tau_{x} G$ overlaps $\left\{x: x_{1}=0, x_{1}=2 \ell\right\}$. Hence the right hand side of $(3.10)$ converges to $c(\rho) / 2 c_{0}$ with $\mathrm{c}$ given in Theorem 1. Since $\sigma_{\Lambda}(G)$ is the expectation of a fixed local function, it converges to $\sigma(G)$, the expression inside the infimum of (2.7). Taking the infimum over $G$ yields the lower bound (2.12).

Acknowledgements HS thanks S.R.S. Varadhan and the Courant Institute for a most friendly hospitality. 


\section{References}

[1] L. van Hove, Phys. Rev. 93, 1374 (1954).

[2] S. Ma, Modern Theory of Critical Phenomena. Benjamin, Reading MA, 1976.

[3] P.C. Hohenberg, P.I. Halperin, Rev. Mod. Phys. 49, 435 (1977).

[4] P.I. Halperin, P.C. Hohenberg, S. Ma, Phys. Rev. Lett. 29, 1548 (1972).

[5] D.P. Landau, Monte Carlo Studies of Critical and Multicritical Phenomena. In: Applications of the Monte Carlo Method, edited by K. Binder, Topics in Current Physics, Vol. 36, p. 93. Springer, Berlin, 1984.

[6] R. Kutner, K. Binder, K.W. Kehr, Phys. Rev. B 28, 1846 (1983).

[7] T. M. Liggett, The Stochastic Evolution of Infinite Systems of Interacting Particles, Lecture Notes in Mathematics Vol. 598. Springer, Berlin, 1978.

[8] H.-O. Georgii, Canonical Gibbs Measures, Lecture Notes in Mathematics Vol. 760. Springer, Berlin, 1979.

[9] I.D. Lawrie, S. Sarbach, Theory of Tricritical Points. In: Phase Transitions and Critical Phenomena, ed. by C. Domb, J.L. Lebowitz, Vol. 9, p. 1. Academic, London, 1984.

[10] R. B. Griffiths, Phys. Rev. B7, 545 (1973).

[11] J.L. Lebowitz, in: Mathematical Problems in Theoretical Physics, edited by G. Dell' Antonio et. al., Lecture Notes in Physics, Vol. 80, p. 68. Springer, Berlin, 1978.

[12] M. Aizenman, Rigorous Study of Critical Behavior. In: Statistical Physics and Dynamical Systems, edited by J. Fritz, A. Jaffe, D. Szász. Birkhäuser, Boston, 1985.

[13] B. Simon, Statistical Mechanics of Lattice Gases, Vol. I. Princeton University Press, Princeton, 1993.

[14] H. Spohn, Large Scale Dynamics of Interacting Particles. Springer, Berlin, 1991. 
[15] F. Rezakhanlou, Comm. Math. Phys. 129, 445 (1990).

[16] S.R.S. Varadhan, Proceedings Taniguchi Symposium, Kyoto, 1990.

[16] J. Quastel, H.T. Yau, Hydrodynamics of Lattice Gas with Random field at Infinite Temperature, in preperation. 